\title{
Formation of branched structure of polyimide macromolecules in the temperatures range below the onset of the thermal destruction
}

\author{
Valentin Svetlichnyi, Iosif Gofman*, Andrey Didenko and Elena Vlasova \\ Institute of Macromolecular Compounds, Russian Academy of Sciences, 31, Bol'shoi str. (V.O.), St. Petersburg, 199004, Russia
}

\begin{abstract}
The film samples of poly-[4,4'-bis(4"-N-phenoxy)biphenyl-sulfone]imide1,3-bis(3',4-dicarboxyphenoxy)benzene obtained both by two-step synthesis and by onestep high temperature synthesis in $\mathrm{N}$-methyl-2-pyrrolidone solution were used to study the formation of the interchain crosslinks in this soluble polyimide in the temperature range of $250-300^{\circ} \mathrm{C}$. Just in this region a final stage of the thermal treatment of different polyimide materials takes place during their processing. A possible crosslink formation mechanism has been put forward to present the adequate explanation of the experimental data obtained in the work. A simple technological way is offered to avoid the unfavourable effect above.
\end{abstract}

\section{Introduction}

The problems concerning the chemical interactions of macrochains of aromatic polyimides (PIs) during the synthesis and subsequent thermal treatments of these polymers is a focus of attention in both polymer and material sciences. The permanent interest to this subject arises from the important role of PI materials in current progress of different branches of modern technics insured by the unique combination of thermal, mechanical, dielectric and other properties of these materials. Under this reason it is extremely important to provide a deep optimization of the protocol of formation processes of these materials. As a rule, these processes include the thermal treatments as a final stage of the PIs processing technologies. Under this reason the questions concerning the PI macrochains' thermally stimulated interactions were analyzed in different scientific works [1-5]. As a rule, these works are devoted to the investigations of the processes which take place at the temperatures above $350-400^{\circ} \mathrm{C}$, i.e. to the interactions of polymer chains during the initial stage of thermal destruction of PIs [1-3]. Indeed, the formation of the interchain crosslinks in these polymers caused by the interaction of the functional groups yet at the initial stages of the destruction seems to be a rather reasonable process. The formation of this type of crosslinks was proved by numerous experimental data [2-5].

But taking into account this mechanism of the formation of "high temperature" crosslinks one should not neglect some possibility of the realization of interchain interactions just in the course of the formation of PIs, at the temperatures less than those of the onset of the thermal destruction processes, namely at $250-300^{\circ} \mathrm{C}$. The possibility of the formation of these "low temperature" crosslinks is supported by the fact of the gradual decrease of the solubility of these polymers in organic solvents after the thermal treatments in this temperature range [2]. Unfortunately this problem has not been properly discussed in the scientific literature up to date in spite of its great importance: the processes of the "structurization" of PIs at these moderate temperatures can significantly affect the properties of the materials especially the solubility and the melt stability, and hence affect their processability.
The new data concerning the formation of the interchain crosslinks in PIs in the temperatures range under consideration $\left(250-300^{\circ} \mathrm{C}\right)$ were obtained during the experiments with the soluble and fusible PIs, namely polyetherimides (PEIs) synthesized by using some tetranuclear aromatic diamines containing so-called bridge groups [6-9].

\section{Experimental}

\section{Starting monomers and solvents}

4,4'-bis(4"-aminophenoxy)biphenylsulfone (98\%, TCI, CAS: 13080-89-2) - diamine BABS, 1,3- bis(3',4-dicarboxyphenoxy) benzene (98\%, Ltd «Tech. Chim. Prom.», Russia) - dianhydride R, phthalic anhydride (99\%, ABCR, CAS: 85-44-9)-PA, toluene (SigmaAldrich, CAS: 24-45-11) were used as received without purification; N-methyl-2-pyrrolidone (97\%, Sigma-Aldrich, CAS: 120-94-5) - MP was dehydrated by refluxing over $\mathrm{CaH}_{2}$ for $6 \mathrm{~h}$ and then distilled under reduced pressure $(30 \mathrm{mmHg})$ at $80^{\circ} \mathrm{C}$.

\section{Polymers}

The objects of our research were the film samples of the PEIs of two structures: PEI-I and PEI-II (Scheme 1). Each of the PEIs studied was synthesized by two methods: the two-step method [6], and the onestage high temperature synthesis in MP solution [8]. The scheme of the process is presented below (Scheme 2).

The formation of the PEIs studied by both methods was conducted via the stage of prepolymers-poly(amic acid)s (PAA) synthesis by

*Correspondence to: Iosif Gofman, Institute of Macromolecular Compounds, Russian Academy of Sciences, 31, Bol'shoi str. (V.O.), St. Petersburg, 199004, Russia, Tel: +7(812)328-8511; E-mail: gofman@imc.macro.ru

Key words: curing, interchain crosslinks, polyimides, thermal destruction

Received: April 05, 2019; Accepted: April 24, 2019; Published: April 26, 2019 
<smiles></smiles>

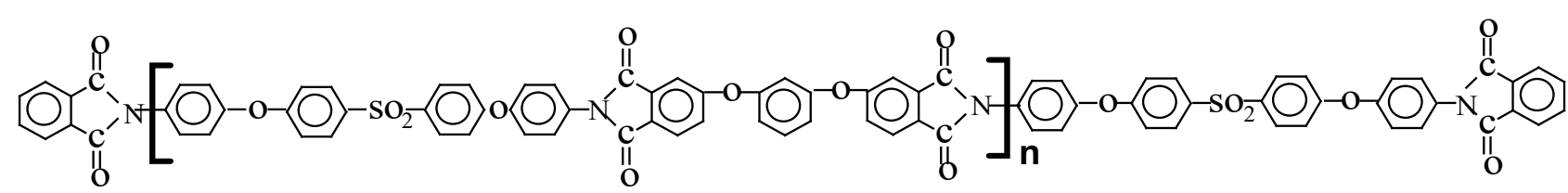

Scheme 1. Structures of polyimides studied in the work.<smiles></smiles><smiles></smiles><smiles>CCCCC(C)C(=O)N1C(=O)c2ccc(Oc3cccc(Oc4ccc(Oc5ccc(S(=O)(=O)c6ccc(Oc7ccc(C)cc7)cc6)cc5)cc4)c3)cc2C1=O</smiles>

Scheme 2. The synthetic route of PI.

solution polycondensation of 1,3- bis(3',4-dicarboxyphenoxy)benzene dianhydride and 4,4'-bis(4"-aminophenoxy)diphenyl sulfone. To prepare the PEI-I the PAA solution (20\%) was obtained by using the equimolar amounts of both monomers (1:1). To obtain the PEIII the molar concentrations ratio of the dianhydride and diamine of 0.99:1.0 was used. To block the excessive amount of amino groups in the polymer chains the phthalic anhydride $(0.02 \mathrm{~mol}$.) was introduced into reaction.

The curing reaction of the obtained PAAs was carried out in two ways: in the solid phase or in solution.

In the first case PAA solution in MP was cast onto clean glass plates and dried in a dry air-flowing thermal chamber at $60^{\circ} \mathrm{C}$ for 6 hours to obtain the PAA films, which were then stepwise cured in vacuum to obtain the totally imidized solvent free PEI films. The protocols of PAA thermal treatment used are presented in table 1.

In the second case (the one-stage method) the imidization of previously synthesized PAAs was conducted in the solution. The water formed during this reaction was removed in the form of azeotrope "toluene-water". This process resulted in the formation of PEI solution in MP, which was then cast onto clean glass plates and dried according to the schedules similar to that of the thermal curing of the PAA films (Table 1). In both cases the thickness of all PEI films formed was $25 \pm 5 \mathrm{mcm}$.

Characterization of the filmsFor the quantitative determination of the concentration of insoluble fraction in the PEI films subjected to the thermal treatments in different conditions, a previously weighed film samples of a mass of $\sim 0.1 \mathrm{~g}$ were inserted into glass flasks with $10 \mathrm{ml}$ of MP. Each sample was kept in the flask in room temperature conditions for $36 \mathrm{~h}$. After this treatment, the solution obtained was filtered through a previously weighed Schott filter, and the precipitate was weighed after drying on the filter at $300^{\circ} \mathrm{C}$ up to the constant weight. The percent amount of insoluble fraction in a PEI film was calculated as the ratio of the weight of the precipitate and that of the starting film.

Intrinsic viscosity values [ $\eta$ ] of the PEIs in MP were determined in accordance with [8].

Mechanical characteristics of the films at room temperature were determined under the uniaxial extension conditions using band-like 
Table 1 Concentrations of insoluble fraction formed upon dissolution in MP of the PEI film samples obtained by using different protocols of synthesis and subsequent heat treatment

\begin{tabular}{|c|c|c|c|c|c|}
\hline No & Polymer & Synthesis method & $\begin{array}{l}\text { Ratio of dianhydride: diamine: PA } \\
\text { (mol) }\end{array}$ & $\begin{array}{l}\text { Heat treatment modes: temperature, } \\
{ }^{\circ} \mathrm{C} \text {, duration }\end{array}$ & Insoluble fraction, $\%$ \\
\hline $\begin{array}{l}1 \\
2 \\
3 \\
3 \\
4 \\
5\end{array}$ & $\begin{array}{l}\text { PEI-I } \\
\text { PEI-I } \\
\text { PEI-II } \\
\text { PEI-II } \\
\text { PEI-I }\end{array}$ & $\begin{array}{l}\text { Single stage } \\
\text { Two-stage (imidization of PAA film) } \\
\text { Single stage } \\
\text { Two-stage (imidization of PAA film) } \\
\text { Two-stage (imidization of PAA film) }\end{array}$ & $\begin{array}{l}1: 1: 0 \\
1: 1: 0 \\
0.99: 1: 0.02 \\
0.99: 1: 0.02 \\
1: 1: 0+\text { PA (1 wt. } \%)\end{array}$ & $\begin{array}{l}\text { a) } 100,200-1 \mathrm{~h}, 300-10 \mathrm{~min} \\
\text { b) } 100,200-1 \mathrm{~h}, 300-1 \mathrm{~h} \\
\text { a) } 100,200-1 \mathrm{~h}, 300-10 \mathrm{~min} . \\
\text { b) } 100,200-1 \mathrm{~h}, 300-1 \mathrm{~h} \\
\text { c) } 100,200,250-1 \mathrm{~h} \\
\text { a) } 100,200-1 \mathrm{~h} ., 300-10 \mathrm{~min} . \\
\text { b) } 100,200-1 \mathrm{~h}, 300-1 \mathrm{~h} \\
\text { d) } 100,200-1 \mathrm{~h}, 300-1 \mathrm{~h}, 380-2 \mathrm{~h} \\
\text { a) } 100,200-1 \mathrm{~h} ., 300-10 \mathrm{~min} . \\
\text { b) } 100,200-1 \mathrm{~h}, 300-1 \mathrm{~h} \\
\text { d) } 100,200-1 \mathrm{~h}, 300-1 \mathrm{~h}, 380-2 \mathrm{~h} \\
\text { b) } 100,200-1 \mathrm{~h}+300-1 \mathrm{~h}\end{array}$ & $\begin{array}{l}12 \\
75 \\
42 \\
76 \\
- \\
0 \\
0 \\
6 \\
0 \\
0 \\
24 \\
0\end{array}$ \\
\hline
\end{tabular}

samples of $2 \mathrm{~mm}$ width and $25 \mathrm{~mm}$ long. Experiments were carried out on AG-100kNX Plus universal mechanical tests system (Shimadzu, Japan). The extension speed was $5 \mathrm{~mm} / \mathrm{min}$.

The same equipment was used to study the stress relaxation processes in the PEI films. These tests were conducted at the temperature $245^{\circ} \mathrm{C}$. The TG $209 \mathrm{~F} 1$ Iris (NETZSCH, Germany) thermal analyzer was used to carry out the thermogravimetric tests (TGA) of $\sim 3 \mathrm{mg}$ samples of PEI films. These specimens were analyzed in $\mathrm{Al}_{2} \mathrm{O}_{3}$ crucibles with a heating speed of $5 \mathrm{deg} / \mathrm{min}$. The temperatures of the thermal destruction onset of the films studied were determined by these tests.

The FTIR spectra of films were recorded using a Vertex-70 FTIR spectrometer (Bruker) equipped with a ZnSe-attenuated total reflectance accessory (PIKE Technologies) at room temperature in a wavelength range $4000-400 \mathrm{~cm}^{-1}$. In the process of collecting attenuated reflectance spectra, we made a correction that takes into account the depth of penetration of the irradiation depending on the wavelength. Raman spectrum was recorded by using a Nicolet iS50R spectrometer (laser $1024 \mathrm{~nm}$ ).

The intermolecular structure of PEI films subjected to the thermal treatments at different temperatures was investigated by wide-angle $\mathrm{X}$-ray diffraction (WAXD) analysis method using the general purpose X-ray diffractometer DRON-8 (Bourevestnik, Inc., Russia) with CuKaradiation $(\lambda=1.54 \AA \hat{)})$ and monochromatization of the incident beam by a Ni-filter. The radiation was directed transversally to the surface of the samples prepared in form of films' stacks of the overall thickness of 1-2 mm.

The film's density, $\rho$, was estimated using the flotation method as described in [10] with a laboratory-made measurement unit. A mixture of toluene and carbon tetrachloride was used to equilibrate the specimens at $20^{\circ} \mathrm{C}$. The mass-spectral thermal analysis (MTA) was conducted by a quadrupole mass-spectrometer TA-QMS 403 C Aëolos (NETZSCH, Germany) coupled with the TG 209 F1 Iris thermal analyzer in which a heating of the samples under investigation was performed.

\section{Results and discussion}

To characterize the processes occurring in PEI-I films during their thermal treatments in the temperatures range up to the onset of the thermal destruction the solubility in MP was estimated of the samples 1 and 2 of this PEI previously subjected to thermal treatments in different conditions (modes a and $\mathrm{b}$, Table 1 ).

The results of these tests (Table 1) show that yet the thermal treatment at $300^{\circ} \mathrm{C}$ provokes a partial loss of the solubility of the polymers prepared both by one-step and two-step methods (samples 1 and 2, respectively). For example a short $(10 \mathrm{~min})$ treatment at $300^{\circ} \mathrm{C}$ leads to a more pronounced decrease of solubility ( $42 \%$ of insoluble material) of films prepared by two-step method (sample 2, thermal treatment mode a) as compared to that prepared by one-step synthesis with the subsequent thermal treatment at the same temperature (12\% of insoluble material, sample 1, thermal treatment mode a). The increase of the duration of films' thermal treatment at the same temperature up to $1 \mathrm{~h}$ causes the increase of the volume of the insoluble fraction up to the same level (75-76\%) for the samples obtained by both methods (samples 1 and 2, thermal treatment mode b).

Basing on these data one can conclude that in both cases some chemical reactions occur during the thermal treatment at $300^{\circ} \mathrm{C}$ that provoke the formation of a crosslinked polymer structure. The intensity of these thermally stimulated reactions presumably depends upon the conditions of the PEI curing process used. The described effect cannot be treated as a result of the initial stage of thermal destruction of the polymer. Indeed, according to the TGA data the thermal destruction process in PEI-I starts at $\sim 400-405^{\circ} \mathrm{C}$ (Figure 1, curve 1 ). These data are in agreement with the results presented in [5].

The conclusion above corresponds well to the results of the MTA of a set of soluble and fusible PEIs containing different bridge groups [5]. This experimental method, very sensitive to some petty variations of the chemical structure of the samples showed that, in the materials studied, the initial stage of the thermal destruction starts only at the temperatures above $360-380^{\circ} \mathrm{C}$ (Figure 2). Thus, it is clear that the cross-linking associated with thermal degradation process could not be realized in the PEIs at temperatures up to $300^{\circ} \mathrm{C}$.

On the other hand, the studied effects cannot be ascribed to the completion of the polymer curing process. This fact testifies the kinetic curve of thermal cyclization of the PAA -precursor of PEI-I, the conversion of the PAA into PEI (Figure 1, curve 2) that demonstrates the full completion of PAA cyclization process up to the range of temperatures examined in our work. The film samples prepared from the PEI solution, obtained via one-step synthesis, were subjected to the additional thermal treatment after their formation that in all cases guarantees the completion of curing process in these films too.

Really, the glass transition temperature of PEI-I is $215^{\circ} \mathrm{C}$ [7], and the thermal treatment of the films during their curing up to the temperatures above the $\mathrm{Tg}$ of the polymer provokes a complete removal of any steric hindrances and insures the completion of the curing process [2].

Thus, the data obtained testify the formation of the insoluble fraction in the PEI films studied in the temperature range in which the curing process is already completed and the thermal destruction does not start yet (Figure 1). It means that some processes of chemical 


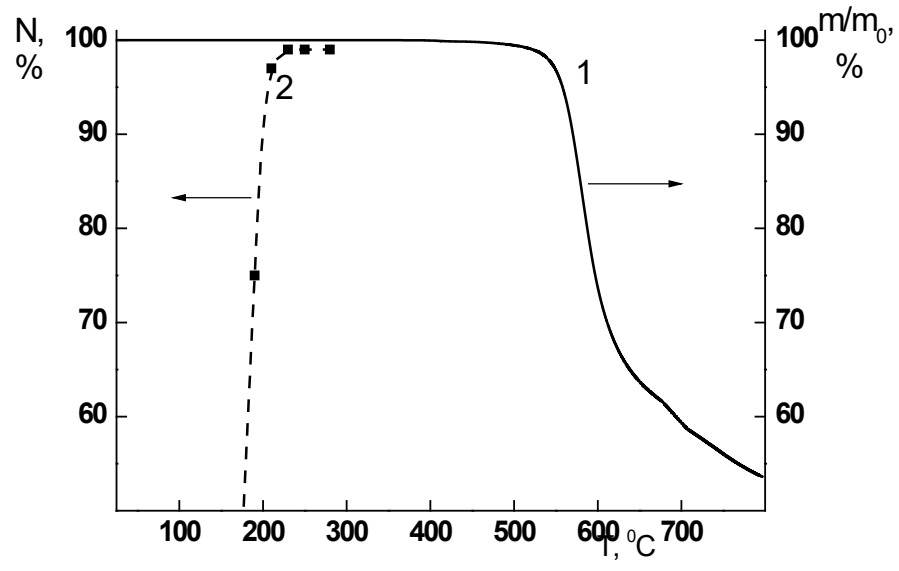

Figure 1. (1) TGA curve of the sample of PEI-I film; (2) kinetic curve of curing process (percent of PAA conversion into PEI vs. temperature) of the PAA to PEI-I: second step of the two-step synthesis.

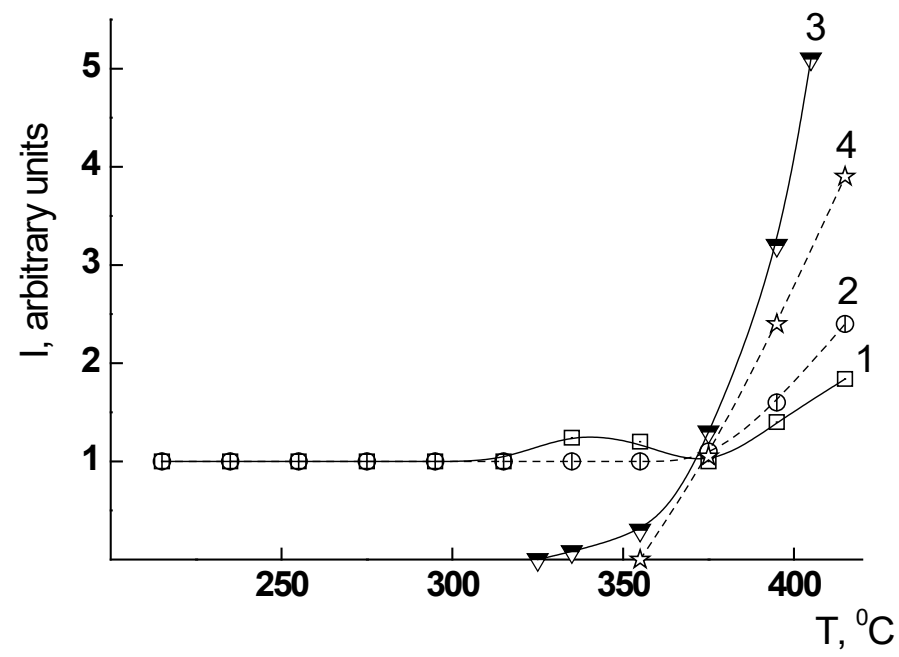

Figure 2. MTA curves for PEI-I. The release curves of the following volatile groups are presented: (1) $\mathrm{H}_{2} \mathrm{O}, \mathrm{M}=18$; (2) $\mathrm{CO}, \mathrm{M}=28$; (3) $\mathrm{SO}_{2}, \mathrm{M}=68$; (4) $\mathrm{OC}_{6} \mathrm{H}_{4} \mathrm{O}, \mathrm{M}=108^{[12]}$.

structurization of polymer chains are developed in the films of the PEI studied during the thermal treatment at the temperature of about $300^{\circ} \mathrm{C}$.

To confirm this conclusion, we have carried out the mechanical tests of the PEI-I films that were previously subjected to the thermal treatments at different temperatures. The films for this investigation were obtained by two-step synthesis, and the temperatures of the termination of their thermal treatments were varied from 250 up to $350^{\circ} \mathrm{C}$. In all cases the treatment's duration was $0.5 \mathrm{~h}$. In these tests the values of the yield stress were measured (Table 2).

These characteristics correlates with the specific energy of the intermolecular interactions in the film materials and is very sensitive to the processes of the variation of polymer structure. A monotonic increase of the yield stress value along with the increase of the temperature of thermal treatment of the films was registered (Table 2). The results of this experiment convincingly certify the formation of some additional intermolecular interactions during the thermal treatment of the PEI films and the increase of amount of these interactions along with the raise of the temperature of treatment.

It should be noted that the mechanical data above may be ascribed not only to the formation of some interactions of chemical nature.
In principle, the same effects could originate in some processes of the physical nature, namely to some physical structurization, some increase in the material's crystallinity, the extent of ordering of the intermolecular structure caused by the thermal treatments. During our investigation special experiments have been undertaken to check the validity of this interpretation of the mechanical results obtained.

The impact of the thermal treatments under study on the features of the intermolecular structure of the films was tested by the WAXD method. The comparison of diffraction pattern of the PEI-I film obtained by the two-step synthesis with the thermal treatment up to $250^{\circ} \mathrm{C}$ (mode $\mathrm{c}$, Table 1 ) and that of the same film subjected to an additional heating at $300^{\circ} \mathrm{C}$ during 1 hour (mode b) shows that this additional heat treatment provoking the substantial decrease in the solubility of film (76\% of insoluble fraction, Table 1) does not cause any variations of the character of the intermoleculat structure of the material (Figure 3). Both diffraction patterns presented in Figure 3 testify of the same completely amorphous structure of the compared films. Only one amourphous halo with the same angle position of the maximal intensity can be seen in both curves.

The same conclusion concerning the absence of any processes of the increase in the extent of ordering of intermolecular structure in course of the films' thermal treatment was drawn from the results of the densitometric tests of PEI films. The density of the film subjected to the thermal treatment at $300^{\circ} \mathrm{C}$ for 1 hour, $\rho_{\exp }$ was $1.359 \mathrm{~g} / \mathrm{cm}^{3}$. While comparing this experimental value of density with the value of Van-der-Vaals density of the polymer $\rho_{\text {calc }}=1.993 \mathrm{~g} / \mathrm{cm}^{3}$ calculated by the atomic increments method [11] the molecular packing coefficient value $k=0.682$ was obtained that testifies the completely amorphous stricture [12] of the heat treated sample of the PEI.

The additional proofs of the formation of the interactions of some chemical nature, the interchains' interactions in the PEI films during the thermal treatments were obtained in the mechanical tests of PEI-I films in the stress relaxation mode. These tests were carried out at the temperature as high as $245^{\circ} \mathrm{C}$ which is 30 degree above the Tg value

Table 2. The yield stress values of PEI-I films samples heat-treated up to different temperatures.

\begin{tabular}{|c|c|c|c|c|c|}
\hline $\mathrm{T},{ }^{\circ} \mathrm{C}$ & 250 & 280 & 300 & 320 & 350 \\
\hline Yield stress, MPa & $110 \pm 2$ & $112 \pm 1$ & $119 \pm 2$ & $121 \pm 2$ & $126 \pm 2$ \\
\hline
\end{tabular}

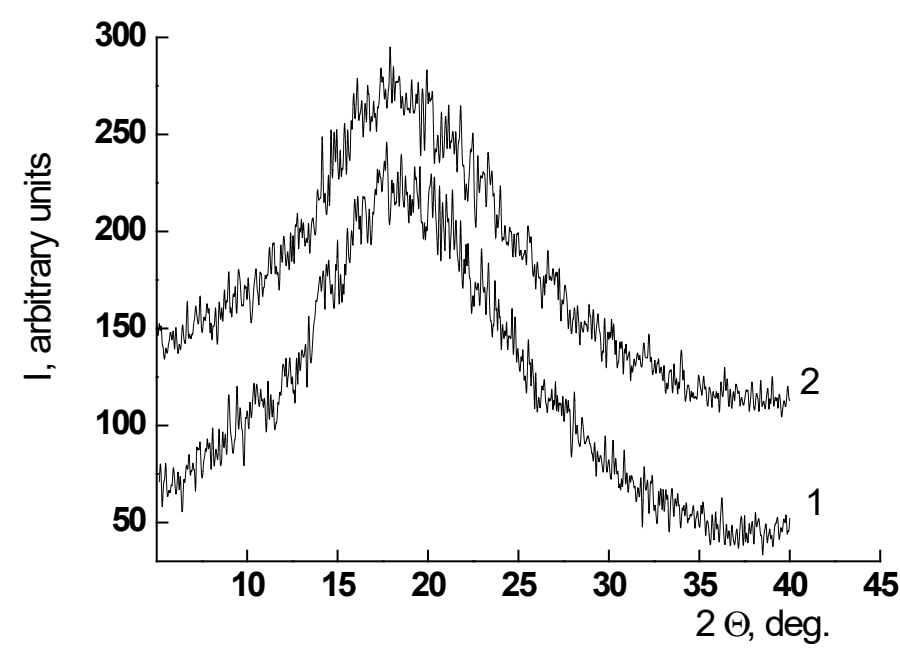

Figure 3. Diffractograms of PEI-I films subjected to different modes of thermal treatment (1) mode a, (2) mode b (Table 1). 
of the polymer. In these conditions we carry out the stress relaxation of the material in the rubbery state. These tests were conducted with the sample extension values of $2.0 \%$, i.e. in the range of the reversible elastic deformations of the materials. The film samples prepared by two-stage synthesis (sample 2, Table 1) were used for these tests.

The results of the tests have shown that the behavior of the films in the stress relaxation conditions (Figure 4) strongly depends upon their thermal pre-history. During the relaxation test of the sample that was subjected to the thermal treatment on the mode $\mathrm{b}$ (Table 1): $1 \mathrm{~h}$ at $300^{\circ} \mathrm{C}$, the asymptotic decrease of the stress was registered up to $\sigma_{\infty}=0.28 \sigma_{\max }$, where $\sigma_{\max }$ is the stress value at the onset of the relaxation process (Figure 4, curve 1). This type of relaxation behavior in the rubbery state is inherent to the crosslinked polymer materials containing a system of chemical intermolecular bonds $[13,14]$. For PEI film that was pre-heated only up to $250^{\circ} \mathrm{C}$ (thermal treatment on the mode $\mathrm{c}$, Table 1) the full stress relaxation takes place up to zero stress value (Figure 4, curve 2). It means that there are no strong chemical intermolecular bonds in the material.

One of the possible reasons for the formation of a branched structure in the process of PIs synthesis during heat treatment to $300^{\circ} \mathrm{C}$ may be the interaction of free amino end groups of polymer chains with the imide cycle and the formation of Schiff base type structures, as it was shown elsewhere by using model structures $[15,16]$. It should be borne in mind that at the initial stage of polycondensation synthesis of PIs a violation of the equimolarity of the reagents is possible (formation of some little excess of free amino groups) not only because of the possible hydrolysis of the anhydride groups, but also because of their inactivation by different impurities [17]. This assumption is consistent with the results obtained while studying the molecular weight distribution of a soluble PI [18]. Thus, macromolecules containing terminal amino groups can be formed in the course of the PI synthesis.

From the above noted, it becomes clear that the structurization processes during the curing of PAA and subsequent heat treatment at $300^{\circ} \mathrm{C}$, are associated precisely with reactions involving free terminal amino groups. It is reasonable to assume (see the scheme below) that the formation of a Schiff base type structure (structure A) is possible during the heat treatment due to a nucleophilic attack of terminal amino group against imide cycle (Scheme 3 ).

In this reaction, the removal of water takes place. This process, exactly, is fixed in the temperature range from $300^{\circ} \mathrm{C}$ by MTA (Figure $2)$. As a result, the formation of the imine bonds $(=\mathrm{C}=\mathrm{N}-)$ can be assumed. The spectroscopic study carried out using IR (Figure 5) and Raman spectroscopy (Figure 6) confirmed the formation of imine bonds when the PEI samples were heated at $300^{\circ} \mathrm{C}$. In the IR spectra of the PEI-I films samples 1 and 2 (heat treatment mode b) a modest peak presents at $1652 \mathrm{~cm}^{-1}$ (Figure 5, curve 2).

This band may be due to the formation of a $\mathrm{C}=\mathrm{N}$ imine bonds. In the IR spectrum of sample, subjected heat treatment in mode $\mathrm{c}$ (final temperature of treatment $-250^{\circ} \mathrm{C}$ ), this band is absent (Figure 5, curve 1). The Raman spectrum of the film heated at $300^{\circ} \mathrm{C}$ (heat treatment mode b) shows the carbonyl band at $1740 \mathrm{~cm}^{-1}$, and a band at $1652 \mathrm{~cm}^{-1}$ (Figure 6); the latter is ascribed to imine $\mathrm{C}=\mathrm{N}$ bond [19].

To obtain the positive proof of the hypothesis above the synthesis of PEI-II samples was specially carried out, in which the terminal amino groups were blocked by phthalimide groups. By this way the formation of aforementioned branched structures was prevented. The corresponding PEI film samples were obtained (Table 1, samples 3 and 4).

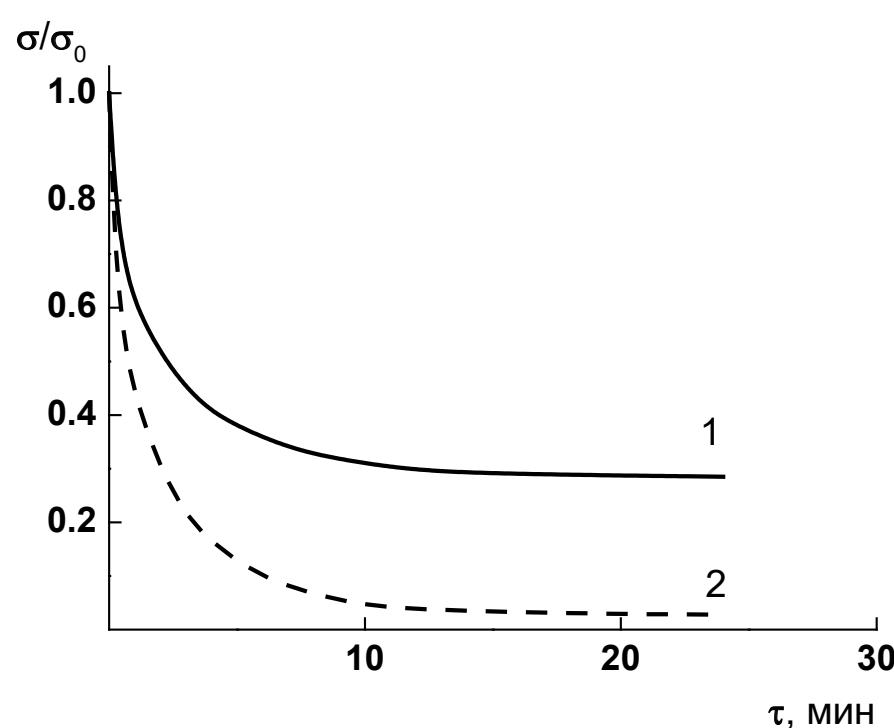

Figure 4. Stress relaxation curves at $245^{\circ} \mathrm{C}$ of the PEI-I films subjected to different modes of thermal treatment: (1) mode a, (2) mode b (Table 1).

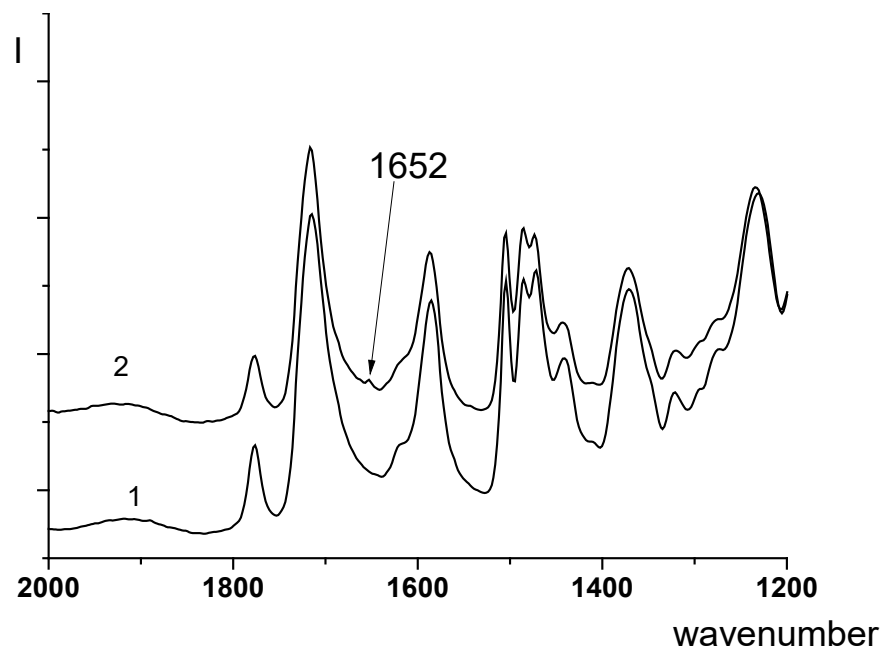

Figure 5. FTIR spectra of PEI-I films (single-stage synthesis): (1) heat treatment mode a; (2) heat treatment mode b (Table 1).

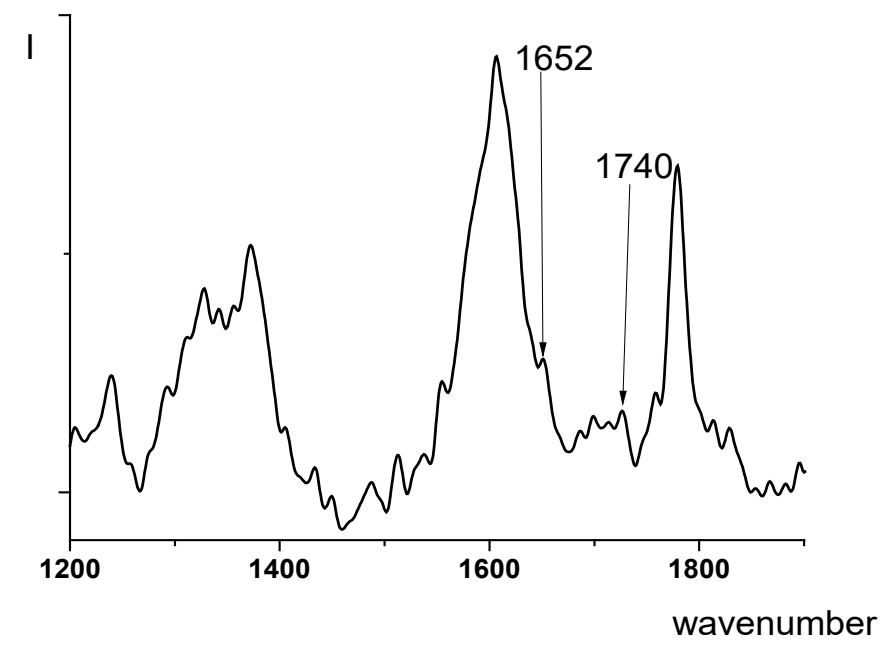

Figure 6. Raman spectrum of PEI-I (single stage-synthesis, heat treatment mode b).A 
<smiles>[R]c1ccc(N2C(=O)OC3(C(=O)N(C)C(=O)C3(C)C)C2=O)cc1</smiles>

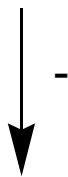<smiles>[R]c1ccc(-n2c(=O)oc(=O)n2C)cc1/N=C1\OC(=O)N(CC)C1=O</smiles>

(A)<smiles>[R]=[R]COc1ccc(S(=O)(=O)c2ccc(OC)cc2)cc1</smiles>

Scheme 3. Scheme of branched structure formation of Schiff base type for PEI-I.

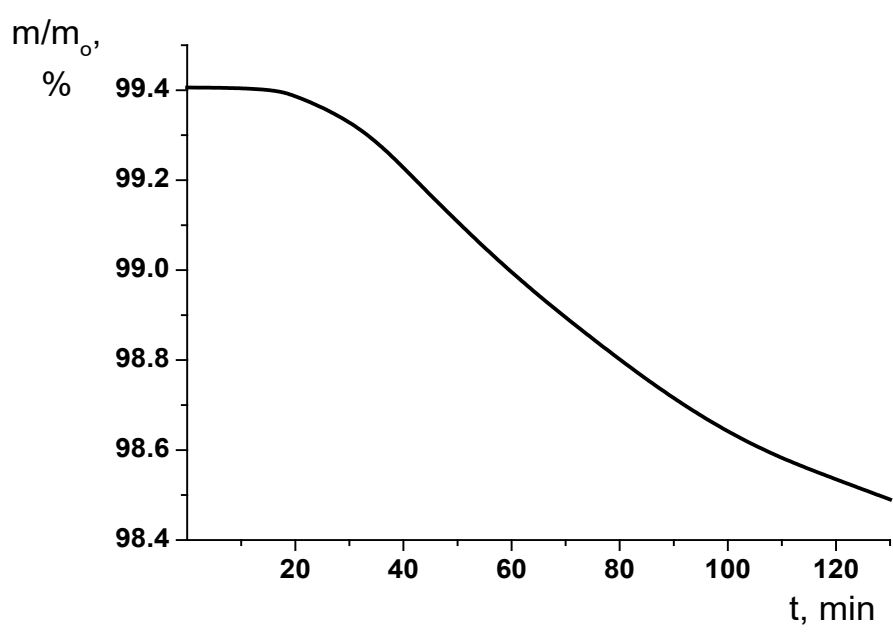

Figure 7. Isothermal TGA curve of PEI-II at $380^{\circ} \mathrm{C}$.
The molecular masses of PEI-II synthesized by this method, calculated from data of sedimentation-diffusion analysis, were found to be within (59-65) $\times 10^{3}$ [20]. Heating of PEI-II films obtained both by single-stage and two-stage methods (samples 3 and 4, Table 1) at $300^{\circ} \mathrm{C}$ for 1 hour (heat treatment mode b) did not lead to a loss of their solubility. That is, the data obtained indicate no formation of a branched structure during the synthesis of PEI with blocked terminal amino groups.

The additional experiments performed in our work have shown that these PEI-II film samples form an insoluble fraction only after a prolonged heating at the temperatures $380-400^{\circ} \mathrm{C}$ (heat treatment mode $\mathrm{d}$, Table 1). In these thermal conditions, the development of the initial stage of thermally stimulated destructive processes takes place as the isothermal TGA data testify (Figure 7), resulting in the formation of interchain crosslinks by radical mechanism during the decomposition of weak chain links [2,9]. The data presented in Table 1 show that the PEI-II films obtained by one-stage method of synthesis 
are more stable against the thermal destruction in these conditions $(6 \%$ of insoluble fraction, sample 3, Table 1) than those obtained by twostage method (24\% of insoluble fraction, sample 4 , Table 1). It means that the concentration of the weak links in the PEI-II films is strongly dependent upon the method of synthesis used: in the sample 4 (twostage synthesis) it exceeds that in the film 3 (single-stage synthesis). The same correlation was shown above while the properties of PEI-I films were discussed (samples 1 and 2, Table 1).

To obtain the final proof of the hypothesis above we have introduced some amount ( $1 \mathrm{wt} . \%$ in relation to dry PAA) of phthalic anhydride (PA) in the solution of previously synthesized PAA, precursor of PEI-I to block the terminal amino groups previously formed in the PAA chains. The solution obtained in such a way was kept under stirring for 1 hour. The PEI-I film formed from this solution retains the full solubility in MP after the heat treatment at $300^{\circ} \mathrm{C}$ for 1 hour (sample 5, Table 1 ). To check the reliability of this, result the experiment was repeated several times. So, this very simple and inexpensive technological approach can prevent the formation of branched structure of a PI material and some uncontrolled variations of properties during its processing.

\section{Conclusions}

The experimental results presented convincingly indicate the possibility of realization of interchain chemical interactions in PIs in the range of temperatures below those of the beginning of thermal destruction of the polymers. Just these interactions result in the formation of cross-linked structures in the pre-destructive range of temperatures.

We paid a close attention to this issue due to the fact that the noted structurization processes occurring at relatively low temperatures (just during the final stage of PIs' synthesis) can have a significant effect on their solubility after synthesis. This peculiarity of the polymers' structure can be important for different areas of their practical use. For example, it can impact on the properties of gas separation membranes, and on the rheological behavior of PEI melts when used as binders for composite materials.

It was shown that the introduction of small amounts (up to 1 wt.\%) of phthalic anhydride (relative to dry PAA) into the solution of the previously formed PAA allows to avoid the process of PI structurization during the thermal curing of PAA and (if it is necessary) the subsequent heat treatment of PI material at the temperatures up to the beginning of thermal decomposition.

\section{Acknowledgments}

Authors acknowledge the help of $\mathrm{T}$ B Kimstach (Moscow department of "Intertech" company) in Raman investigations and of $\mathrm{V}$ K Lavrentiev (Institute of Macromolecular Compounds, RAS) in X-ray examination of the films studied.

\section{References}

1. Buhler KU (1978) Spezialplaste. Akademie Verlag. Berlin, pp: 1015.

2. Bessonov MI, Koton MM, Kudryavtsev VV, Laius LA (1987) Polyimides - thermally stable polymers. Consultants Bureau, New York, pp: 318.

3. Smirnova VE, Gofman IV, Maritcheva TA, Yudin VE, Eto K, et al. (2007) The effect of different orientations in rigid rod polyimide films on the graphitized products. Carbon 45: 839 .

4. Inagaki M, Hishiyama Y, Kaburagi Y (1994) Effect of heating rate during carbonization on graphitization of carbon films derived from aromatic polyimides. Carbon 32: 637.

5. Svetlichnyi VM, Kudryavtsev VV (2003) Polyimides and the problem of formation of modern composite materials. Polym Sci B 45: 140.

6. Koton MM, Svetlichnyi VM, Kudryavtsev VV, Smirnova VY, Maricheva TA, et al (1980) Polyimides with ether-sulphone groups on the amino-component. Polym Sci U.S.S.R. 22: 1163

7. Svetlichnyi VM, Zhukova TI, Kudriavtsev VV, Gubanova GN, Yudin VE, et al. (1995) Aromatic polyetherimides as promising fusible film binders. Polym Eng Sci 35: 1321.

8. Nesterov VV, Kudryavtsev VV, Svetlichnyi VM, Gazdina NV, Bel'nikevich NG, et al (1997) Study of soluble poly(amic acid)s and poly(ester imide)s by methods of exclusion liquid chromatography. Polym Sci A 39: 953.

9. Koton MM, Panov YN, Svetlichnyi VM, Shibaev LA (1989) In Polyimides: Materials, Chemistry and Characterization, ed by Feger C, Khojasteh MM, McGrath JE. Elsevier Science Publishers BV, Amsterdam, pp: 403.

10. Pientka Z, Brozova L, Pulyalina A, Goikhman M, Podeshvo I, et al. (2013) Synthesis and Characterization of Polybenzoxazinone and its Prepolymer Using Gas Separation. Macromol Chem and Phys 214: 2867.

11. Askadskii AA (2005) Computational materials science of polymers. Cambridge International Science Publishing.

12. Askadskii AA (2003) Lectures of physico-chemistry of polymers. Nova Science Publishers, New York.

13. Tager AA (1980) Physical Chemistry of Polymers. Central Books Ltd, London.

14. Hu W (2013) Polymer Physics. A Molecular Approach. Springer Verlag, Wien.

15. Saini AK, Carlin CM, Patterson HH (1993) Confirmation of the presence of imine bonds in thermally cured polyimides. J Polym Sci A Polym Chem 31: 2751.

16. Koning C, Delmotte A, Larno P, Van Mele B (1998) Influence of polymerization conditions on melt crystallization of partially aliphatic polyimides. Polymer 39: 3697.

17. Coppinger GM (1954) Preparations of N,N-Dimethylamides. J Am Chem Soc 76: 1372

18. Vasilenko NA, Akhmet'eva YI, Sviridov YB, Berendyayev VI, Rogozhkina YD, et al. (1991) Soluble polyimides based on 4,4'-diaminotriphenylamine. Synthesis, molecular mass characteristics and solution properties. Polym Sci U.S.S.R. 33: 1439.

19. Saini AK, Carlin CM, Patterson HH (1992) Spectroscopic studies of new model compounds for poly[N,N'-bis(phenoxyphenyl)pyromellitimide]. J Polym Sci A Polym Chem 30: 419.

20. Okatova OV, Didenko AL, Svetlichnyi VM, Pavlov GM (2016) Hydrodynamic, molecular, and conformational characteristics of poly[1,3-bis(3',4-dicarboxyphenoxy) benzene 4,4'-bis(4"-N-phenoxy)-diphenylsulfone]imide in solutions. Polym Sci A 58: 12 .

Copyright: (C2019 Svetlichnyi V. This is an open-access article distributed under the terms of the Creative Commons Attribution License, which permits unrestricted use, distribution, and reproduction in any medium, provided the original author and source are credited. 